\title{
Influence of Pinning Effects on the Electrochemical Formation of Silver Patterns in Agarose-Containing Sols and Gels
}

\author{
M. A. Pasquale, ${ }^{\dagger, *}$ G. P. Saracco, ${ }^{\dagger}$ S. L. Marchiano, ${ }^{\dagger}$ and A. J. Arvia $*, \dagger$ \\ Instituto de Investigaciones Fisicoquímicas Teóricas y Aplicadas (INIFTA, CONICET-UNLP), Sucursal 4, \\ Casilla de Correo 16, 1900 La Plata, Argentina, and Facultad de Ciencias Fisicomatemáticas e Ingeniería, \\ Pontificia Universidad Católica, C1107AFD Buenos Aires, Argentina
}

Received: April 1, 2005; In Final Form: July 19, 2005

\begin{abstract}
The formation of silver patterns via electrolysis from aqueous silver sulfate $+x \% \mathrm{w} / \mathrm{v}$ agarose sol and gel media, with and without supporting electrolyte, in a quasi-two-dimensional (2D) cylindrical cell at room temperature, is utilized as a reference system to investigate the complexity of pinning effects. From pattern morphology and electrochemical data, both delocalized and localized pinning in the bulk dominate the drift of the growth front, depending on the concentration of agarose in the heterogeneous media. Delocalized pinning results from mobile, small agarose aggregates at the growth front and from their accumulation by the front drift. For gels, localized pinning comes from their own percolated structure. A depinning/pinning transition is observed in going from sols to gels. The relative contribution of diffusion and advection in mass-transportcontrolled silver electrodeposition depends on the plating bath composition. On the other hand, silver ion attachment to the cathode appears to be interfered with by some screening caused by weakly adsorbed, mobile agarose aggregates at the metal surface without slowing down the rate of the electron-transfer step at the cathode. Their relative contribution of a delocalized, localized pinning and screening effect to a great extent determines the morphology and transition in the growth mode of silver patterns in both media. The analysis of charge and current transients and the corresponding silver pattern morphologies for open and dense radial patterns is made. Results are qualitatively simulated with a novel, rather simple cellular automaton algorithm.
\end{abstract}

\section{Introduction}

Interfacial phenomena in both homogeneous and heterogeneous environments, involving either an immobile or mobile front, are of a great importance in both $l i f e^{1-3}$ and material sciences. ${ }^{3-5}$ The behavior of these phenomena, particularly for mobile fronts, depends on the degree of disorder in the environment that affects its propagation velocity. For a mobile front, the propagation velocity results from the balance between the local resistance of the environment against propagation and the driven force acting upon the interface. ${ }^{6,7}$ The timeindependent local resistance is produced by a quenched noise, i.e., a localized pinning interfering in the interface propagation. Then, the interface becomes either pinned or depinned when either the local resistance or the driven force, respectively, dominates the force balance at the interface. This implies the possible existence of pinning/depinning transitions related to the dynamics of the interface. ${ }^{7}$ This type of phenomenon, among others, has been observed in the crystallization of proteins, mass transfer processes in natural and synthetic porous media ${ }^{8-14}$ and polymer electrolytes,${ }^{8-9}$ paper wetting experiments, ${ }^{15}$ propagation of the burning front, ${ }^{16}$ growth of bacterial colonies, ${ }^{17,18}$ rupture of lines in paper sheets, ${ }^{19}$ solid growth fronts, ${ }^{20}$ electrochemical formation of solid phases, ${ }^{21}$ tumoral growth mechanisms, ${ }^{22}$ and their growth and spreading. ${ }^{23-25}$

The diversity of processes involved in the above phenomena suggests the possibility to investigate time-dependent pinning

\footnotetext{
* Corresponding author. E-mail: ajarvia@inifta.unlp.edu.ar.

† Instituto de Investigaciones Fisicoquímicas Teóricas y Aplicadas.

\$Facultad de Ciencias Fisicomatemáticas e Ingeniería, Pontificia Universidad Católica.
}

effects that may occur, for instance, for front propagation in colloidal media. In these media, either localized or delocalized obstacles emerging from the specific size and velocity distribution of polymeric aggregates in sols and gels should interfere in the dynamics of the interface. ${ }^{26}$

In this work, the electrochemical growth of quasi-twodimensional (2D) silver patterns from agarose-containing sol and gel plating baths is utilized as a test process to distinguish between localized and delocalized pinning using current transient data and pattern morphology. The overall process is under mass transport control, as the electron-transfer step for silver electrodeposition becomes one of the fastest electrochemical processes. ${ }^{27-29}$ However, in agarose-containing media, silver electrodeposition is affected by a delocalized screening at the metal/medium interface, its net effect being a slight decrease of the cathode surface area for allocation of silver at the metal lattice. This delocalized screening, together with the Brownian motion of small agarose aggregates, partakes in the formation of silver patterns. On the other hand, particularly for sols, the drift of the growth front itself promotes a delocalized pinning due to the change in the distribution of agarose aggregates at the interface. For gels, localized pinning due to the percolated structure of the medium is of considerable importance.

Two scenarios are proposed to describe silver pattern formation and current transient data in sol and gel media. In the absence of supporting electrolyte, both delocalized pinning for sols and localized pinning for gels play a key role in the process. Conversely, in the presence of supporting electrolyte, pattern formation from sol and gel media is subjected to both delocalized and localized pinning and pinning from the accumulation of small agarose aggregates pushed outward by the growth front. 


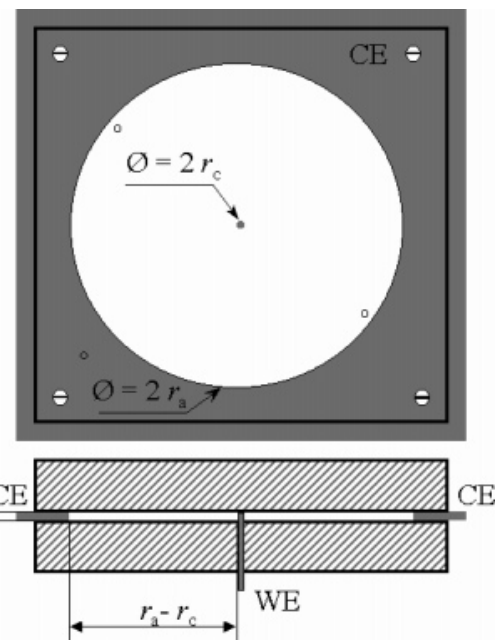

Figure 1. Top and lateral views of a typical quasi-2D horizontal circular electrochemical cell; $r_{\mathrm{c}}$ and $r_{\mathrm{a}}$ are the working electrode (cathode) (WE) and counter electrode (anode) (CE) radius, respectively; $l_{\mathrm{s}}=0.025 \mathrm{~cm}$ is the thickness of the solution layer in the cell.

Pinning favors the formation of silver fractal patterns with thick branching. This morphology is consistent with a random noise in the current transient that appears when dense branching is produced. For the above scenarios, the pattern morphologies and current transients resulting from sol and gel media are simulated with a rather simple, novel cellular automaton algorithm, where pinning at the silver/environment interface is mimicked, taking into account the influence of the sol and gel structure on the process.

\section{Experimental Section}

The silver patterns were grown from different plating baths utilizing a quasi-2D cylindrical electrochemical cell. The latter consisted of top and bottom parallel flat glass plates separated by the distance $l_{\mathrm{s}}=0.025 \mathrm{~cm}$ and a concentric arrangement of electrodes with a central silver wire cathode ( $99.9 \%$ purity, radius $r_{\mathrm{c}}=0.20 \mathrm{~cm}$ ) and a silver ring anode (radius $r_{\mathrm{a}}=5 \mathrm{~cm}$ ) (Figure 1). Occasionally, a comparable electrochemical cell with a plane plate electrode design described elsewhere ${ }^{26}$ was utilized.

Aqueous 0.014 and $0.024 \mathrm{M}$ silver sulfate $+x \% \mathrm{w} / \mathrm{v}$ agarose $(0 \leq x \leq 0.6)$ plating baths, either supporting electrolyte-free or with the addition of $0.50 \mathrm{M}$ potassium sulfate $+0.01 \mathrm{M}$ sulfuric acid as supporting electrolyte to make the contribution of migration of silver ions negligible, were utilized. Plating baths were prepared from analytical reagent-grade chemicals and Milli-Q water. Agarose-containing plating baths prepared in situ $^{26}$ were sufficiently stable to produce reproducible, reliable data. ${ }^{30,31}$

Cathodic polarization curves were recorded at $0.100-0.300$ $\mathrm{V} / \mathrm{s}$ to minimize the cathode area variation and surface roughness effects. The plane plate cell design was useful for avoiding the spreading of silver electrodeposits and for attempting to distinguish a possible effect of agarose on the rate of the electron-transfer process at the cathode.

Potentiostatic silver electrodeposition was run by applying different cathode-to-anode potential differences $\left(\Delta E_{\mathrm{c}-\mathrm{a}}\right)$. As the anodic overpotential $\left(\eta_{\mathrm{a}}\right)$ for silver electrodissolution was negligible because of the large surface area of the anode and the high exchange current density $\left(j_{0}\right)$ value of the $\mathrm{Ag}^{+} / \mathrm{Ag}$ electrode (negligible activation overpotential, $0.1 \leq j_{0} \leq 20 \mathrm{~A}$ $\left.\mathrm{cm}^{-2}\right),{ }^{27-29}$ values of $\Delta E_{\mathrm{c}-\mathrm{a}}$ were referred to the potential of the anode. The cathodic concentration overpotential $\left(\eta_{\mathrm{c}}\right)$ was obtained after the corresponding ohmic drop correction $\eta_{\mathrm{ohm}}=$
$I_{\mathrm{c}} R_{\mathrm{c}}$, i.e., $\eta_{\mathrm{c}}=\Delta E_{\mathrm{c}-\mathrm{a}}-I_{\mathrm{c}} R_{\mathrm{c}}$, where $R_{\mathrm{c}}$ is the ohmic resistance between the cathode and the anode. Values of $\eta_{\mathrm{c}}$ covering the range $-0.650 \leq \eta_{\mathrm{c}} \leq-0.170 \mathrm{~V}$ were taken as the concentration overpotential at the silver cathode. ${ }^{26,32}$

Silver growth patterns were sequentially pictured at different time $(t)$, and simultaneously, the cathodic current $\left(I_{\mathrm{c}}\right)$ and charge $\left(Q_{\mathrm{c}}\right)$ transients were recorded. The pattern morphology and the maximum radial displacement $\left(R_{\mathrm{M}}\right)$ of the growing front were derived from images obtained with a Zeiss Stemi 200C stereoscopic microscope coupled to either a digital camera Cannon G5 Powershot or a video camera connected to a computer equipped with an image analyzer. Occasionally, a sequence of photographs was taken using a Contax camera 167 MT coupled to the microscope. All runs were made at room temperature $(298 \pm 1 \mathrm{~K})$. The fractal dimension $\left(D_{\mathrm{F}}\right)$ of silver patterns was determined from the $\log Q_{\mathrm{c}}$ versus $\log R_{\mathrm{M}}$ plots. ${ }^{33,34}$

\section{Results}

3.1. Polarization Curves. The polarization curves were plotted as $j_{\mathrm{c}}$, the cathodic current density referred to the initial cathode area, versus either $\Delta E_{\mathrm{c}-\mathrm{a}}$, the cathode-to-anode potential difference, or $\eta_{\mathrm{c}}$. Results from aqueous solutions with and without the addition of supporting electrolyte are depicted in Figure 2a. For the same $c_{\mathrm{Ag}+}{ }^{o}$, the cathodic limiting current density $\left(j_{\mathrm{L}}\right)$ in the supporting electrolyte-containing solution is close to one-half the value found for the supporting electrolytefree solution, as expected. ${ }^{35}$ Accordingly, values of $\eta_{\mathrm{c}}$ were selected in the range for $j_{\mathrm{c}}<j_{\mathrm{L}}$, where the concentration of silver ions at the cathode surface $\left(c_{\mathrm{Ag}+}\right)$ is either smaller than that in the bulk $\left(c_{\mathrm{Ag}+}{ }^{o}\right)$ or in the range of $\eta_{\mathrm{c}}$ pertaining to the limiting current density where $c_{\mathrm{Ag}+}=0$. The $\eta_{\mathrm{c}}$ versus $j_{\mathrm{c}}$ plots obey the Nernst equation, expressed as $\eta_{\mathrm{c}}=\eta_{\mathrm{c}}\left(j_{\mathrm{c}}, j_{\mathrm{L}}\right)$, for a concentration cell without liquid junction, ${ }^{35}$ as expected for a mass-transport-controlled process.

The same Nernstian behavior is observed for the polarization curves resulting from agarose-containing plating baths (curve $1^{\prime}$ in Figure $2 \mathrm{a}$ and $2^{\prime}$ in Figure $2 \mathrm{~b}$ ). The decrease in about $10 \%$ of the cathodic limiting current density in going from $x=0$ to $x=0.60$ can be attributed to a maximal screening of the cathode by the presence of agarose.

3.2. Current Transients. 3.2.1. Supporting Electrolyte-Free Plating Baths. The current transients run for $\eta_{\mathrm{c}}=-0.170 \mathrm{~V}$ (Figure 3a) first exhibit a relatively fast decrease in $I_{\mathrm{c}}$ to reach a minimum value at the time $t_{\mathrm{m}}$. The value of $t_{\mathrm{m}}$ decreases and the current for $t<t_{\mathrm{m}}$ diminishes faster in going from aqueous solutions to sol and gel plating baths, respectively. Later, for $t$ $>t_{\mathrm{m}}$, the value of $I_{\mathrm{c}}$ gradually increases because of the continuous increase in the cathode surface area, although as $x$ is increased, the slope of the current transients decreases. The $Q_{\mathrm{c}}$ versus $t$ plots (Figure $3 \mathrm{~b}$ ) from either free or agarosecontaining media, for $t<50 \mathrm{~s}$, exhibit a linear increase of $Q_{\mathrm{c}}$, and later, after a poorly defined inflection point, the positive slopes of the plots increase with $t$, as expected for metal electrodeposition on a cylindrical cathode.

3.2.2. Supporting Electrolyte-Containing Plating Baths. In the absence of agarose, the current transients run at $\eta_{\mathrm{c}}=-0.650$ $\mathrm{V}$ (Figure $4 \mathrm{a}$ ) first show a decrease in $I_{\mathrm{c}}$ to a minimum value for $t_{\mathrm{m}} \cong 20 \mathrm{~s}$ and, subsequently, an increase almost linearly with $t$. The initial fast decrease of $I_{\mathrm{c}}$ is principally related to the relaxation of the diffusion layer, ${ }^{36}$ whereas its further increase is due to the simultaneous increase in the cathode surface area and the contribution of a macroscopic flow that partakes in the formation of dense radial electrodeposits. ${ }^{37,38}$

In general, for sols and gels, current transients (Figure 4a) show almost the same initial decay of $I_{\mathrm{c}}$ as for $x=0$, followed 

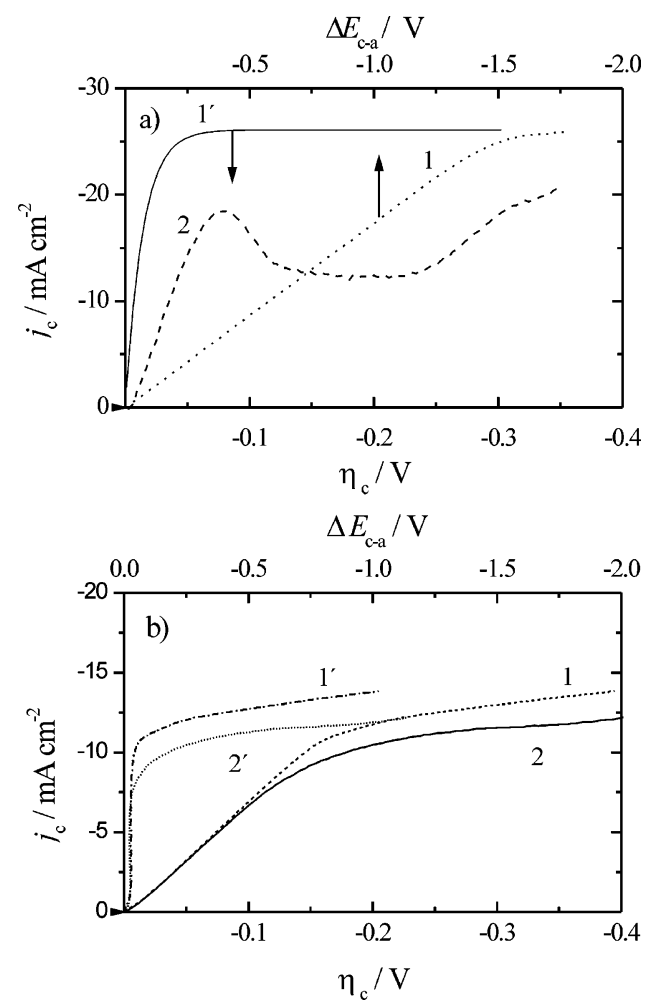

Figure 2. Cathodic polarization curves run at $0.100 \mathrm{~V} / \mathrm{s}$ and $298 \mathrm{~K}$. (a) Agarose-free solutions. $0.024 \mathrm{M}$ silver sulfate without ohmic drop correction (curve 1) and after ohmic drop correction (curve 1'). 0.024 $\mathrm{M}$ silver sulfate $+0.5 \mathrm{M}$ sodium sulfate $+0.01 \mathrm{M}$ sulfuric acid without ohmic drop correction (curve 2). This curve shows first a current hump, mainly due to the relaxation of the diffusion layer, and a cathodic limiting current in the range between -0.125 and $-0.200 \mathrm{~V}$ that corresponds to a pseudosteady limiting current at a cathode surface area still close to the initial one. Subsequently, a further increase in current due to the increase in the cathode surface area can be observed. It should be noted that the limiting current in (1') is close to twice the value in (2), as expected. (b) Cathodic polarization curve from agarosefree $0.024 \mathrm{M}$ silver sulfate $+0.5 \mathrm{M}$ sodium sulfate $+0.01 \mathrm{M}$ sulfuric acid. No ohmic drop correction (curve 1) and after ohmic drop correction (curve $1^{\prime}$ ). Cathodic polarization curve from $0.024 \mathrm{M}$ silver sulfate $+0.5 \mathrm{M}$ sodium sulfate $+0.01 \mathrm{M}$ sulfuric acid $+0.60 \% \mathrm{w} / \mathrm{v}$ agarose. No ohmic drop correction (curve 2) and after ohmic drop correction (curve $2^{\prime}$ ).

by an increase for $t>t_{\mathrm{m}}$. This ascending portion of the $I_{\mathrm{c}}$ versus $t$ plot becomes smaller and tends to disappear as $x$ is increased. Subsequently, a stepwise decrease of $I_{\mathrm{c}}$ is recorded.

For $x=0.05$ (sol), the constant current range recorded between $t \cong 125$ and $t \cong 300$ s suggests a balance between two opposite effects, the increase in the cathode surface area favoring silver electrodeposition and the partial hindrance of the process by the environment. However, for $t>300 \mathrm{~s}$, the quasilinear increase in $I_{\mathrm{c}}$ with $t$ indicates a prevalence of the former effect. For $x=0.20(\mathrm{gel})$, the initial decay of $I_{\mathrm{c}}(t<50 \mathrm{~s})$ is followed by a current build up from $t \cong 50 \mathrm{~s}$ to $t \cong 200 \mathrm{~s}$ and, later, a slow stepwise decay to attain a constant value of $I_{\mathrm{c}}$. For $x=$ 0.35 and $t \geq 200 \mathrm{~s}$, stochastic current outbursts are superimposed to the current transient, their intensity decreasing with $t$. Finally, for $t>1000 \mathrm{~s}$, an almost quasisteady value of $I_{\mathrm{c}}$ is attained. For $x=0.60$, the same behavior is observed, except that the current decay starts earlier.

The slope of the corresponding $Q_{\mathrm{c}}$ versus $t$ plots (Figure $4 \mathrm{~b}$ ) decreases as $x$ is increased, and approaches zero for the highest values of $x$. These results show that the higher the value of $x$, the greater the hindrance of the process by the presence of agarose. Therefore, the pinning-free process for $x=0$ turns
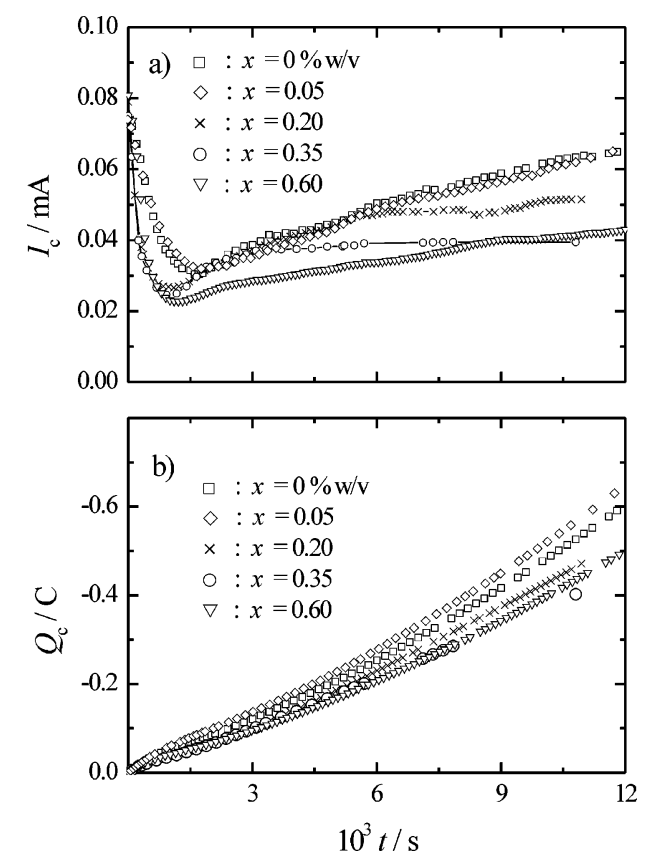

Figure 3. Current transient data at $\eta_{\mathrm{c}}=-0.170 \mathrm{~V}$ from $0.024 \mathrm{M}$ silver sulfate $+x$ agarose. $(\square) x=0 ;(\diamond) x=0.05 ;(\times) x=0.20 ;(\bigcirc)$ $x=0.35 ;(\nabla) x=0.60 ; 298 \mathrm{~K}$. (a) Potentiostatic cathodic current transients. (b) $Q_{\mathrm{c}}$ vs $t$ plots.
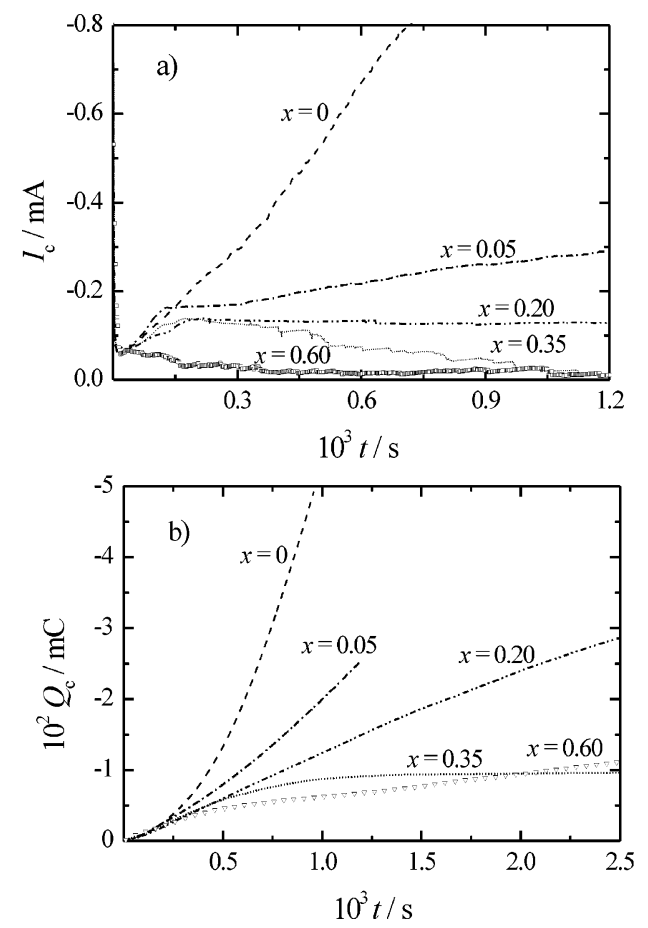

Figure 4. Current transient data. (a) $0.014 \mathrm{M}$ silver sulfate $+0.50 \mathrm{M}$ sodium sulfate $+0.01 \mathrm{M}$ sulfuric acid $+x \% \mathrm{w} / \mathrm{v}$ agarose for $\eta_{\mathrm{c}}=$ $-0.650 \mathrm{~V}$ at $298 \mathrm{~K}$. (b) $Q_{\mathrm{c}}$ vs $t$ plots. The corresponding values of $x$ are indicated in the figure.

into a process under pinning for $x=0.60$. Correspondingly, in the range $0.05 \leq x \leq 0.20$, a depinning/pinning transition takes place.

3.3. Silver Pattern Morphology. 3.3.1. Agarose-Free Plating Solutions. In general, silver patterns consist of a first, relatively compact silver layer comprising the cathodic charge density referred to the initial cathode area $q_{\mathrm{c}}=-1.10 \mathrm{mC} \mathrm{cm}^{-2}$, i.e., about 6800 silver atom monolayers. ${ }^{29,39}$ Subsequently, depending on the solution composition and value of $\eta_{\mathrm{c}}$, different branched morphologies are observed (Figure 5). 
a)
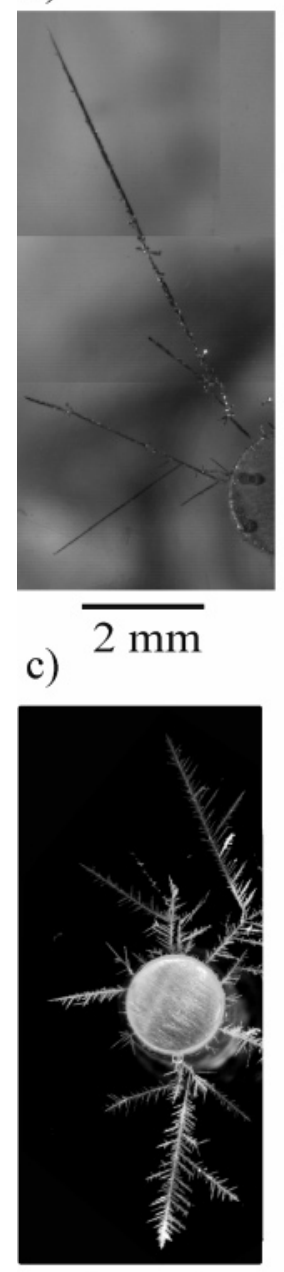

$\overline{2 \mathrm{~mm}}$ b)

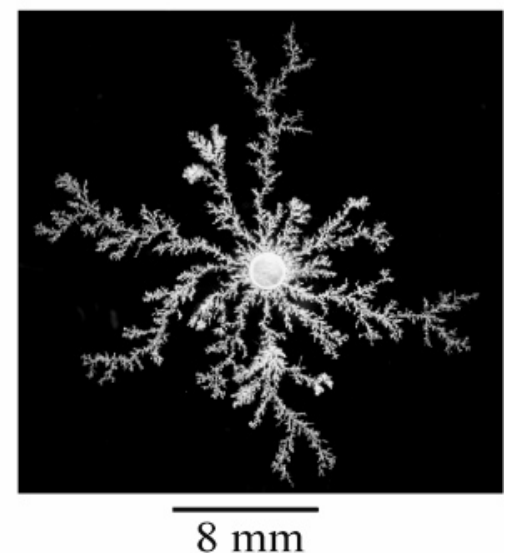

d)

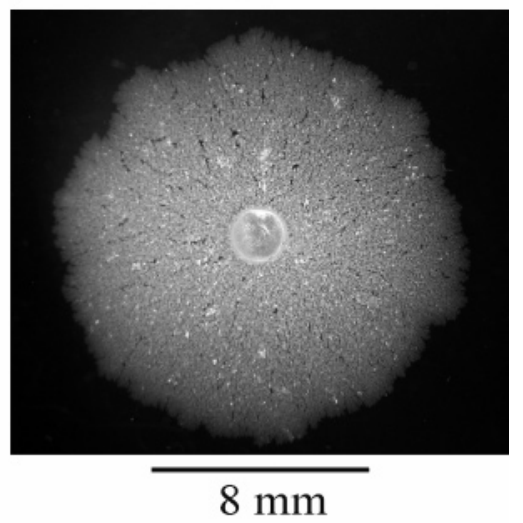

Figure 5. Silver growth patterns obtained in the absence of agarose in the plating bath, at $298 \mathrm{~K}$. (a) $0.024 \mathrm{M}$ silver sulfate; $\eta_{\mathrm{c}}=-0.170$ $\mathrm{V} ; t=5400 \mathrm{~s}$. (b) $0.024 \mathrm{M}$ silver sulfate; $\eta_{\mathrm{c}}=-0.650 \mathrm{~V} ; t=1200 \mathrm{~s}$. (c) $0.014 \mathrm{M}$ silver sulfate $+0.5 \mathrm{M}$ sodium sulfate $+0.01 \mathrm{M}$ sulfuric acid; $\eta_{\mathrm{c}}=-0.170 \mathrm{~V} ; t=6600 \mathrm{~s}$. (d) $0.014 \mathrm{M}$ silver sulfate $+0.5 \mathrm{M}$ sodium sulfate $+0.01 \mathrm{M}$ sulfuric acid; $\eta_{\mathrm{c}}=-0.650 \mathrm{~V} ; t=780 \mathrm{~s}$.

In the absence of supporting electrolyte and $\eta_{\mathrm{c}}=-0.170 \mathrm{~V}$, the pattern (Figure 5a) displays a relatively small number of radial growing domains with side branching. For $t>2000 \mathrm{~s}$, the pattern is dominated by needlelike structures (whiskers), and for $t \cong 3 \mathrm{~h}$, these whiskers become thicker, and randomly distributed specks on stems and side branching, mostly merging at either 60 or $120^{\circ}$ with respect to the needle stem direction, can be seen. On the other hand, for $\eta_{\mathrm{c}}=-0.650 \mathrm{~V}$, i.e., under limiting current conditions (Figure 5b), an open, rather thick branching with tip splitting is then produced.

In the presence of supporting electrolyte and $\eta_{\mathrm{c}}=-0.170$ $\mathrm{V}$, the pattern (Figure $5 \mathrm{c}$ ) consists of dendrites with stems decorated by side branching mostly merging at $60^{\circ}$ with respect to the stem direction. For $\eta_{\mathrm{c}}=-0.650 \mathrm{~V}$ (Figure $5 \mathrm{~d}$ ), a dense radial quasicylindrical pattern is formed. These morphologies are essentially maintained for both $c_{\mathrm{Ag}+}{ }^{\circ}=0.028$ and 0.048 $\mathrm{M}$, although for the latter, a thicker branching is produced.

For both with and without the presence of supporting electrolyte, a linear maximum electrodeposit radius $\left(R_{\mathrm{M}}\right)$ versus $t$ plot is obeyed (Figure $6 \mathrm{a}, \mathrm{b}$, data for $x=0$ ), i.e., the maximum radial velocity $\left(V_{\mathrm{M}}\right)$ of the growth front becomes constant, although the value of $V_{\mathrm{M}}$ becomes considerably greater for the supporting electrolyte-containing solution.
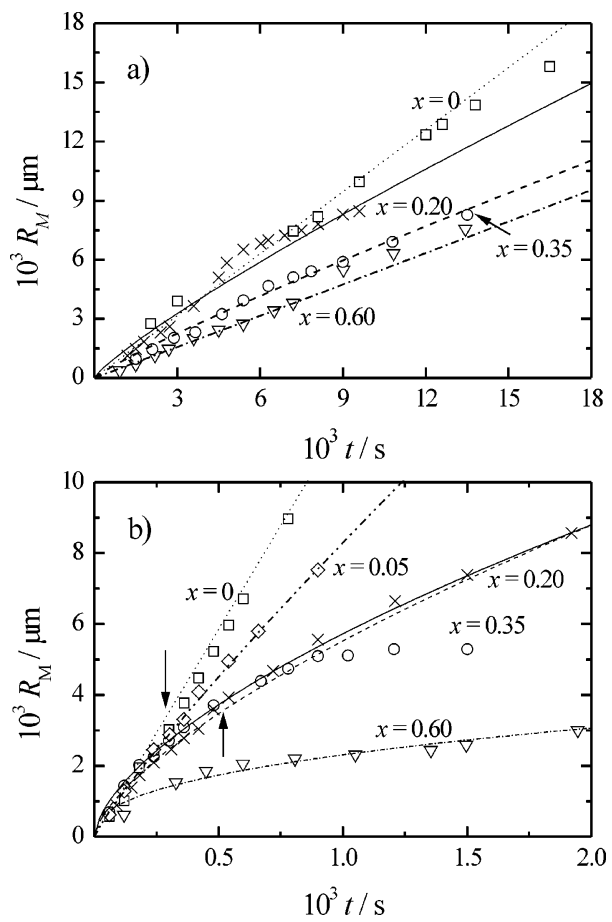

Figure 6. Plots of $R_{\mathrm{M}}$ vs $t$. Traces are calculated with the function $R_{\mathrm{M}}$ $=A t^{d}$. (a) Data from silver patterns obtained from $0.024 \mathrm{M}$ silver sulfate $+x \% \mathrm{w} / \mathrm{v}$ agarose for $\eta_{\mathrm{c}}=-0.170 \mathrm{~V}$ at $298 \mathrm{~K} ;(\square) x=0, A=V_{\mathrm{M}}$ $=1.1 \mu \mathrm{m} \mathrm{s}^{-1}, d=1.0 ;(\times) x=0.20, A=3.6, d=0.85 ;(\mathrm{O}) x=0.35$, $A=1.8, d=0.89 ;(\nabla) x=0.60, A=0.48, d=1.0$. (b) Data from silver patterns produced from $0.014 \mathrm{M}$ silver sulfate $+0.50 \mathrm{M}$ sodium sulfate $+0.01 \mathrm{M}$ sulfuric acid $+x \% \mathrm{w} / \mathrm{v}$ agarose for $\eta_{\mathrm{c}}=-0.650 \mathrm{~V}$ at $298 \mathrm{~K}$. (口) $x=0, A=V_{\mathrm{M}}=11.7 \mu \mathrm{m} \mathrm{s}^{-1}, d=1.0 ;(\diamond) x=0.05$, $A=19, d=0.88 ;(\times) x=0.20, A=54, d=0.67$; (O) $x=0.35, A$ $=79, d=0.62 ;(\nabla) x=0.60, A=147, d=0.39$. Arrows indicate the morphology transition range.

3.3.2. Supporting Electrolyte-Free, Agarose-Containing Plating Baths. In agarose-containing baths, for $\eta_{\mathrm{c}}=-0.170 \mathrm{~V}$, the silver patterns (Figure 7) show an increase in tip splitting and denser morphology as $x$ is increased. Patterns resulting from sols and gels (Figure 7c,d) exhibit some local morphology transitions, the faster their appearance, the larger the value of $x$. For gels, the growth mode transition (Figure $7 \mathrm{c}, \mathrm{d}$ ) occurs for $t>1 \mathrm{~h}$.

For sols and gels, the $R_{\mathrm{M}}$ versus $t$ plots (Figure 6) can be fitted with $R_{\mathrm{M}}=A t^{d}$, the exponent $d$ being in the range $0.85-$ 1 , without showing a particular dependence on $x$. Correspondingly, the value of $V_{\mathrm{M}}$, derived from the $R_{\mathrm{M}}$ versus $t$ plots, tends to decrease with $x$ (Figure 6a). It should be noted, however, that within certain ranges of $t$, the $R_{\mathrm{M}}$ versus $t$ plots show inflections that correlate to local transitions in the growth mode, such as those seen in Figure 7c,d. As can be seen after the growth mode transition, those inflections are consistent with a balance between the decrease in the velocity of the front drift over the largest portion of the front periphery due to pinning and the faster drifts at the rest of the front periphery. Then, only a small number of branch tips continue growing and thickening (Figure 7c,d). Correspondingly, the apparent density of these electrodeposits, defined as the ratio between the silver mass resulting from $Q_{\mathrm{c}}$ and the apparent volume of the electrodeposit, estimated from $R_{\mathrm{M}}$, increases in going from sols to gels (Figure 8). The fractal dimension of patterns from gels is $D_{\mathrm{F}, \mathrm{Ag}}=1.6 \pm 0.1$ for $2 \mathrm{D}$ space.

3.3.3. Supporting Electrolyte, Agarose-Containing Plating Baths. Patterns resulting at $\eta_{\mathrm{c}}=-0.650 \mathrm{~V}$ from sols and gels (Figure 9) exhibit a dense radial morphology accompanied by 
a)

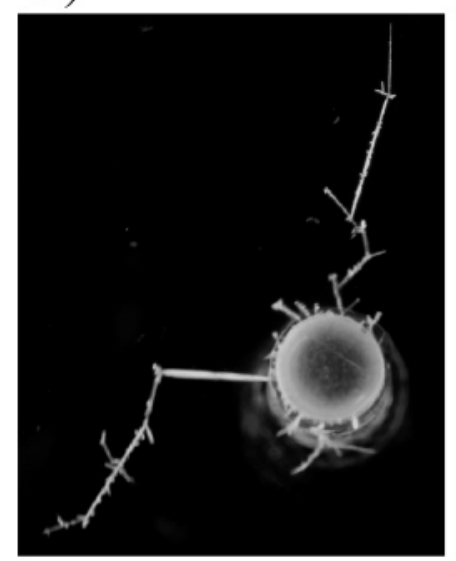

c)

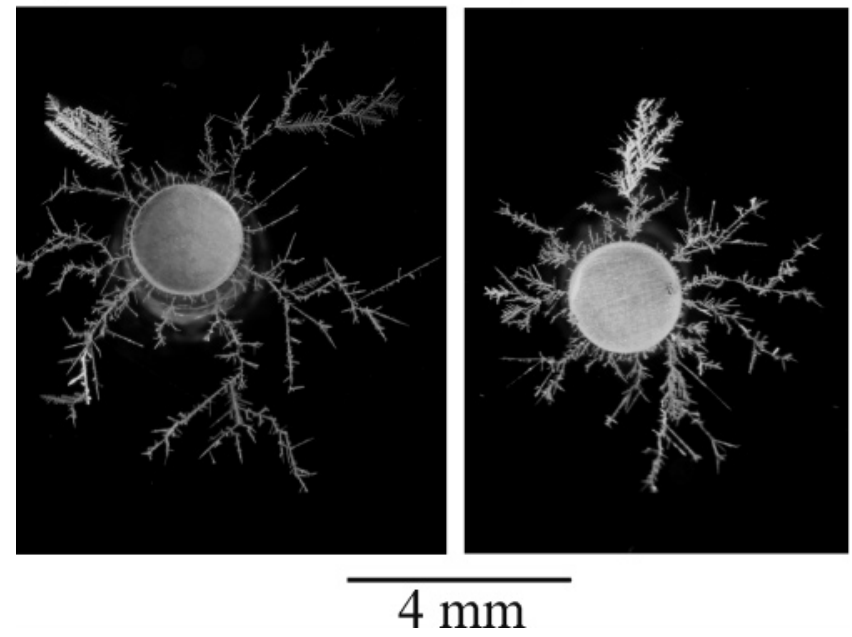

Figure 7. Silver patterns obtained at $\eta_{\mathrm{c}}=-0.170 \mathrm{~V}$ in $0.024 \mathrm{M}$ silver sulfate $+x \% \mathrm{w} / \mathrm{v}$ agarose for $t=5400 \mathrm{~s} ; 298 \mathrm{~K}$. (a) $x=0.05$; (b) $x$ $=0.2 ;$ (c) $x=0.35 ;$ (d) $x=0.60$.

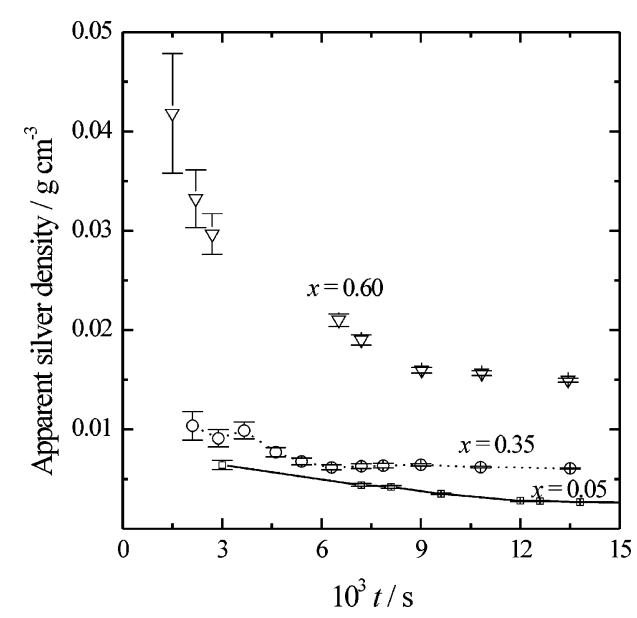

Figure 8. Plots of the apparent density of silver electrodeposits vs $t$. Data from runs made at $\eta_{\mathrm{c}}=-0.170 \mathrm{~V}$ in aqueous $0.024 \mathrm{M}$ silver sulfate $+x \% \mathrm{w} / \mathrm{v}$ agarose; $298 \mathrm{~K} .(\square) x=0.05 ;(\bigcirc) x=0.35 ;(\nabla) x$ $=0.60$. Error bars are indicated.

local transitions in the growth mode from dense radial to a more open dense radial branching. This occurs when both $Q_{\mathrm{c}}$ and $\langle R\rangle$, the average silver pattern radius, exceed certain critical values. For $x=0.05$ (Figure 9a), this change occurs for $Q_{\mathrm{c}} \cong$ $-50 \mathrm{mC}$ and $\langle R\rangle \cong 0.35 \mathrm{~cm}$. The pattern morphology after the transition to some extent resembles that produced from gels a)

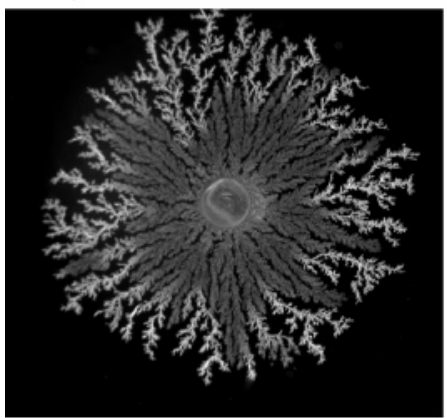

$$
\overline{5 \mathrm{~mm}}
$$

c)

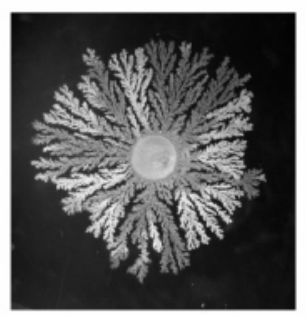

$\overline{5 \mathrm{~mm}}$ b)

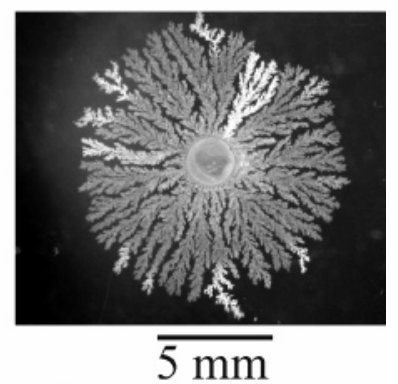

d)

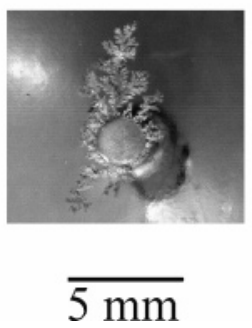

Figure 9. Silver patterns obtained from $0.014 \mathrm{M}$ silver sulfate +0.5 $\mathrm{M}$ sodium sulfate $+0.01 \mathrm{M}$ sulfuric acid $+x \% \mathrm{w} / \mathrm{v}$ agarose for $\eta_{\mathrm{c}}=$ $-0.650 \mathrm{~V} ; t=900 \mathrm{~s} ; 298 \mathrm{~K}$. (a) $x=0.05$; (b) $x=0.20$; (c) $x=0.35$; (d) $x=0.60$.

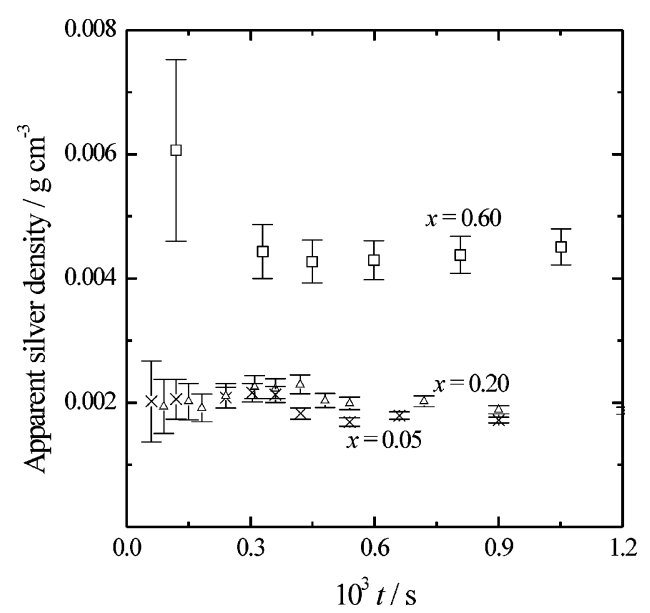

Figure 10. Plot of the apparent density of silver electrodeposits vs $t$. Data obtained from $0.014 \mathrm{M}$ silver sulfate +0.5 sodium sulfate + $0.01 \mathrm{M}$ sulfuric acid $+x \% \mathrm{w} / \mathrm{v}$ agarose for $\eta_{\mathrm{c}}=-0.650 \mathrm{~V}$ at $298 \mathrm{~K}$. $(\times) x=0.05 ;(\triangle) x=0.20 ;(\square) x=0.60$. Error bars are indicated.

(Figure 9c,d). For $x=0.60$, the pattern radial symmetry disappears (Figure 9d).

For sols and gels, at least in the range $0 \leq t \leq 2000 \mathrm{~s}$, the slope of the $R_{\mathrm{M}}$ versus $t$ plots obeys the $R_{\mathrm{M}}=A t^{d}$ relationship (Figure 6b), in this case, the corresponding value of $d$ decreasing with $x$. For sols, the inflection in the $R_{\mathrm{M}}$ versus $t$ plot (see arrows in Figure 6b) coincides with the morphology transition illustrated in Figure 9, which should be related to the accumulation of mobile agarose aggregates pushed outward by the growth front drift. For gels, at longer $t$, a constant value of $R_{\mathrm{M}}$ is presumably attained. The apparent density of these silver patterns (Figure 9) increases with $x$ via branch thickening (Figure 10).

3.4. Current Outbursts and Pattern Morphology. To investigate further aspects of current outbursts overlapping current transients when the electroformation of silver patterns 

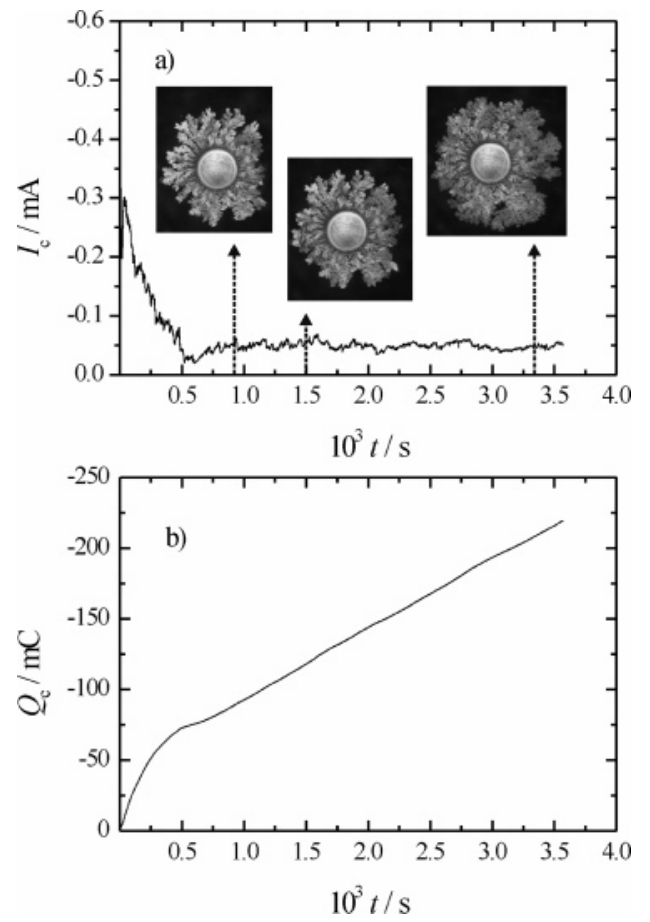

Figure 11. (a) Potentiostatic current transient for silver electrodeposition from $0.024 \mathrm{M}$ silver sulfate $+0.60 \% \mathrm{w} / \mathrm{v}$ agarose for $\eta_{\mathrm{c}}=$ $-0.650 \mathrm{~V}$ at $298 \mathrm{~K}$. Silver growth patterns at different $t$ indicated by the arrows. From $t>50 \mathrm{~s}$ to $t \cong 500 \mathrm{~s}$, the rate of the electrochemical process is hindered by localized pinning due to the percolated structure of the gelled bath. Later, the current decreases to a minimum and for $1000 \leq t \leq 1500 \mathrm{~s}$, although the size of the electrodeposit increases, it seems that the cathode surface area remains almost constant. At this stage, pinning resulting from the accumulation of agarose aggregates at the growth front is evident. For $t>1600 \mathrm{~s}$, the filling of holes and interbranch spaces by electrodepositing silver ions can be noticed. Simultaneously, the average current is almost constant and the intensity of the random noise tends to decrease. (b) $Q_{\mathrm{c}}$ vs $t$ plot from the same experiment. The inflection in the curve corresponds to the commencement of the filling process.

is driven by both migration and diffusion, experiments were made utilizing supporting electrolyte-free aqueous $0.024 \mathrm{M}$ silver sulfate $+0.6 \% \mathrm{w} / \mathrm{v}$ agarose plating bath, at $\eta_{\mathrm{c}}=-0.650$ $\mathrm{V}$. In this case, dense branching patterns are formed (Figure 11a). Random current outbursts appear after having reached the vicinity of a maximum in the current and they remain in the rest of the current transient. In the time window $50 \leq t \leq 500$ $\mathrm{s}$, the value of $I_{\mathrm{c}}$ decreases again to reach a second minimum value at $t \cong 500 \mathrm{~s}$, which can be related to obstacles present in the gel structure to some extent hindering the front drift. Subsequently, in the range $500 \leq t \leq 1000 \mathrm{~s}$, the average current slightly increases because of the increase in the cathode surface area, and for $t>1500 \mathrm{~s}$, the increase in the pattern size is accompanied by the electrodeposition of silver at interbranch channels and holes, leading to a more compact electrodeposit. The beginning of the filling process coincides with the inflection point in the averaged $Q_{\mathrm{c}}$ versus $t$ plot (Figure 11b). Afterward, the growth process continues at an almost constant rate determined by the diffusion of silver ions at holes, as seen from the pattern images (Figure 11a). The time required for the current outburst triggering depends on the driven force, as seen from data shown in Figure 12 for $\eta_{\mathrm{c}}=-0.270 \mathrm{~V}$. In this case, a longer time is required for current outburst triggering, i.e., to overcome the local agarose aggregate resistance. This experiment also confirms the absence of random noise at the ascending portion of the current transient.

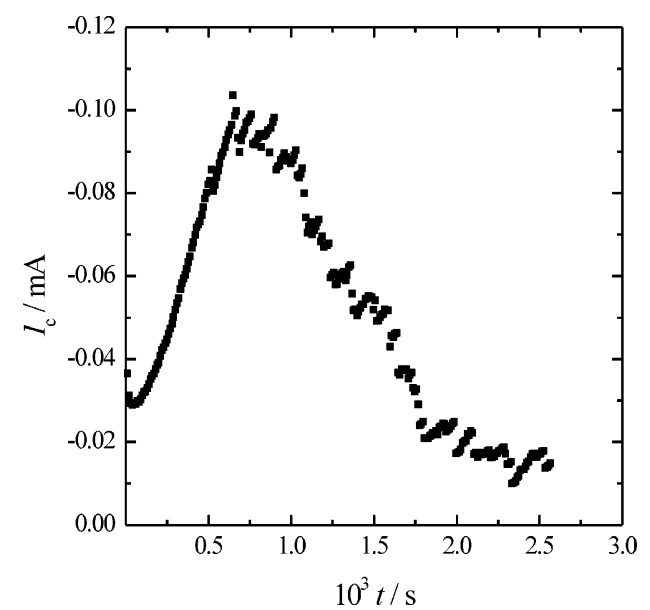

Figure 12. Current transient run at $\eta_{\mathrm{c}}=-0.300 \mathrm{~V}$ from $0.014 \mathrm{M}$ silver sulfate $+0.50 \mathrm{M}$ sodium sulfate $+0.01 \mathrm{M}$ sulfuric acid $+0.35 \%$ $\mathrm{w} / \mathrm{v}$ agarose. The random noise commences in the neighbor of the current maximum at about $500 \mathrm{~s}$.
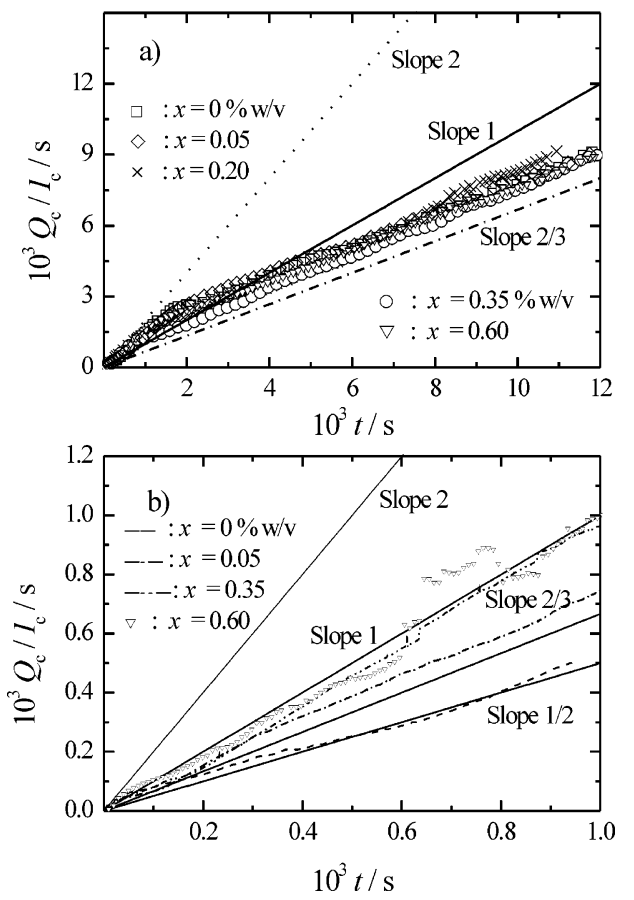

Figure 13. Plots of $Q_{\mathrm{d}} I_{\mathrm{c}}$ vs $t$ for silver electrodeposition at $\eta_{\mathrm{c}}=-0.625$ $\mathrm{V}$ and $298 \mathrm{~K}$. (a) $0.014 \mathrm{M}$ silver sulfate $+x \% \mathrm{w} / \mathrm{v}$ agarose; (b) 0.014 $\mathrm{M}$ silver sulfate $+0.50 \mathrm{M}$ sodium sulfate $+0.01 \mathrm{M}$ sulfuric acid + $x \% \mathrm{w} / \mathrm{v}$ agarose. Values of $x$ are indicated in the figure. Reference straight lines correspond to plane plate electrode geometry. Slope 2 is derived for pure diffusion under constant cathode surface area. Slope 1 is related to either advection or free convection under constant cathode surface area. Slope $2 / 3$ is derived for diffusion under a linear increase in cathode surface area with time. Slope $1 / 2$ is assigned to a process under advection and linear increase in the cathode area with time.

3.5. Mass Transport Processes in the Bulk. The effect of the increase in the cathode surface area in the cylindrical cell that appears in the $I_{\mathrm{c}}$ versus $t$ plots can be minimized by plotting $Q_{\mathrm{c}} / I_{\mathrm{c}}$ versus $t$. These plots can be utilized to attempt to distinguish mass transport contributions to silver growth pattern formation, such as diffusion and advection. ${ }^{26,40}$

For $x=0$, in the absence of supporting electrolyte, current transient data plotted as $Q_{\mathrm{c}} / I_{\mathrm{c}}$ versus $t$ (Figure 13a) asymptotically approach a straight line with slope 2 for $t<1000 \mathrm{~s}$; later, the slope decreases, and for $t \geq 2500 \mathrm{~s}$, data approach the straight line with the slope $2 / 3$. These results correspond to a process dominated by diffusion with negligible change in the 
cathode area for $t \rightarrow 0$ and with linear increase in the electrode surface area with $t$ for $t>2500 \mathrm{~s}$. The largest departure of data from the slope $2 / 3$ appears for $t \cong 1500 \mathrm{~s}$ and $x=0$. It occurs when advection sets in, as has been recently demonstrated for the growth of silver electrodeposits in quasi-2D cells. ${ }^{41}$ Plots of data from sols and gels behave in a rather similar way, except that the experimental data lie closer to the straight line of slope $2 / 3$ and the influence of advection is considerably diminished.

The above behavior of the $Q_{\mathrm{c}} / I_{\mathrm{c}}$ versus $t$ plots, which are associated with open branching patterns, can be explained by considering that the electrochemical process undergoes principally at radial stem tips. From the electrochemical standpoint, these tips can be described as constant capture cross-section hemispheres. ${ }^{29}$ Then, the solution of Fick's equation for each tip, in terms of current, is of the form ${ }^{36}$

$$
I_{\mathrm{dt}}=\Sigma\left(I_{\mathrm{dp}}+z_{i} F D_{i} S c_{\mathrm{i}}^{o} / r_{\mathrm{t}}\right)
$$

where $I_{\mathrm{dt}}$ is the experimental migration-diffusion current, which is the sum of diffusion currents at uncorrelated tips (no tip diffusion layer overlapping); $I_{\mathrm{dp}}$ is the diffusion current at a plane plate; $z_{i}$ is the charge of the reacting ion $i ; F$ is the Faraday; $D_{i}$ is the diffusion coefficient of $i$; $S$ is the cathode surface area; and $r_{\mathrm{t}}$ is the radius of the tip, respectively. Then, for uncorrelated tips with a constant capture area, the second term becomes $t$-independent. Accordingly, the slope of the $Q_{\mathrm{c}} / I_{\mathrm{c}}$ versus $t$ plots should be determined by the first term of eq 1 , and the tendency of data to follow the straight line with slope $2 / 3$ for $t>2500 \mathrm{~s}$ (Figure 11a) can be explained.

In the presence of supporting electrolyte, current transient data plotted as $Q_{\mathrm{c}} / I_{\mathrm{c}}$ versus $t$ (Figure $11 \mathrm{~b}$ ) approach the straight line with slope 1 for $0 \leq t \leq 50 \mathrm{~s}$. In this case, the contribution of advection (slope $1 / 2$ ) is enhanced by the fast electrodeposition rates attained in these experiments, yielding dense radial silver patterns (Figure 5d). Data from these sols and gels (Figure 11b) asymptotically approach the linear plot with slope 1 for $t \rightarrow 0$, whereas for $25 \leq t \leq 150 \mathrm{~s}$, data approach the straight line with slope 0.5 , but for $t>200 \mathrm{~s}$, data lie between the straight lines with slopes ranging from $2 / 3$ to 1 , depending on the time window and composition of the plating bath. The slope 1 line can be related to a diffusion-advection process with constant area, whereas the slope $2 / 3$ corresponds to a diffusion process with a linear increase in the surface area with $t$.

\section{Discussion}

4.1. Some Characteristics of Agarose Sols and Gels and the Silver Electrodeposition Test Reaction. The experimental data indicate that the composition of the silver plating baths plays a relevant role in determining the branching density and the branch radial growth rate and morphology of silver patterns produced in quasi-2D electrochemical cells. Therefore, to advance a model qualitatively accounting for the formation of silver patterns of different morphologies in the quasi-2D cylindrical cell and their corresponding charge transients, it is convenient to remember some specific features of the different agarose-containing plating bath used in this work.

The structure of agarose sols consists of domains of mobile aggregates covering a wide range of molecular weight distributions, whereas gels can be described as percolated complex structures comprising large, almost immobile agarose domains, and channels with a large number of small mobile aggregates distributed in there. ${ }^{26,31}$ The size distribution function of agarose aggregates has been determined by using photographic data from confocal laser scanning microscopy. ${ }^{26}$ The corresponding data fulfill the Korcak empirical law. ${ }^{33}$ Accordingly, for $c_{\mathrm{aga}}=0.60 \%$ $\mathrm{w} / \mathrm{v}$, in the absence of supporting electrolyte, the fractal dimension of the gel $D_{\mathrm{F}, \text { gel }} \cong 2.6 \pm 0.1$ was obtained, as expected for a percolation process under chemical bonding control $^{42}$ and anisotropic drift rates for the silver front. The value of $D_{\mathrm{F} \text {,gel }}$ coincides with those that have been derived from the average roughness exponent calculated from different paper wetting experiments, ${ }^{15}$ the scaling exponent of rupture lines and wetting fronts of filter papers, ${ }^{19}$ and directed percolated depinning models. ${ }^{43-45}$ Then, the structure of agarose gels to some extent resembles the fiber and pore structure and spacing in filter papers. The difference between filter papers and agarose gels comes from the mobility of agarose aggregates, the smaller their size the larger their mobility, and the elasticity of the agarose gel that makes these systems more complicated than conventional inhomogeneous porous materials.

The mechanism of silver electrodeposition on silver in aqueous environments involves: (i) the transport of silver ions from the bulk of the solution to the electrochemical interface, (ii) the electron-transfer step at the solid electrode, and (iii) a rearrangement of adsorbates to assist silver atom attachment to the metal lattice. ${ }^{46,47}$ According to the experimental results, it is clear that the presence of agarose in the environment has a remarkable influence on the transport of silver ions, but it has practically no disturbing effect on the second step, as the cathodic process obeys the Nernstian behavior. This is consistent with the low degree of surface coverage by agarose of the silver cathode $^{31}$ and the low adsorption enthalpy of $\mathrm{OH}$-containing compounds at a number of metal/colloid interfaces. ${ }^{48}$ However, the accumulation of small agarose aggregates at the electrochemical interface, which is assisted by the growth front drift, would produce a certain degree of screening at the cathode for the reactant silver ions that would affect the third step of the reaction pathway. Therefore, the screening effect would be equivalent to a certain loss of cathode area for silver electrodeposition. This effect can be accounted for in modeling the process by properly selecting the corresponding silver attachment probability.

Let us consider now the influence of agarose on the mass transport rate-determining step. For constant driven force, the silver electrodeposition rate increases nearly 1 order of magnitude in going from supporting electrolyte-free to supporting electrolyte-containing solutions, and the presence of agarose produces at least two types of $t$-independent pinning, one which dominates for sols, is a delocalized pinning, and another, which dominates for gels, is a localized pinning due to the percolated structure of the environment. Both effects can be distinguished by their dependence on $x$ and the occurrence of a depinning/ pinning transition in going from aqueous solutions to sols and gels.

A $t$-dependent pinning comes out from the outward displacement by the front drift of mobile agarose aggregates that are accumulated at the outer plane of the interface. This $x$-dependent accumulation of agarose aggregates there promotes a local sol/ gel transition and, correspondingly, introduces an additional resistance to pattern formation. As the silver phase first grows through low resistance channels of the colloidal structure, this $t$-dependent pinning interferes only after a certain time from $t$ $=0$, which depends on $x$ and the silver electrodeposition rate.

The cooperative action of pinnings determines the relative contributions of diffusion and advection to the mass-transportdependent kinetics of the process and pattern morphology as well. Thus, when pinnings brake the drift of the growing front, silver growth at interbranch spaces and holes becomes favored. 
The filling process implies the reaccommodation of agarose aggregates to leave free sites for electrodepositing silver ions. For gels, as $x$ is increased, all pinning and screening described above contribute to hinder the electrochemical process almost completely.

4.2. Silver Pattern Morphology and Pinning Effects. In the absence of supporting electrolyte and constant driven force, the pattern morphology gradually changes from a small number of needlelike branches to a larger number of denser branches as $x$ is increased. In this case, for sols, the main pinning comes from the mobile agarose aggregates, whereas for gels, it is mainly due to their percolated structure, as concluded from 3.3.2.

In the presence of supporting electrolyte, silver patterns change from dense radial to irregular open branching in going from agarose-free solution to gels. In these experiments, the heterogeneous structure of the environment at the interfacial region is changed by the drift of the growth front. This complicated temporal behavior is responsible for the random noise superimposed to the silver electrodeposition current. In this case, apart from those pinnings described above for sols, when $R_{\mathrm{M}}$ reaches a certain critical value that depends on $x$, a net morphology transition is observed (Figure 9a). It occurs when a sufficient amount of agarose accumulates in a ring around the growth front, favoring the local sol/gel transition described in 3.3.3. The thickness $\delta_{\mathrm{r}}$, of this agarose ring can be estimated from the amount of agarose that is displaced from the average volume occupied by the silver electrodeposit of critical average radius $\left\langle R_{\mathrm{c}}\right\rangle$. Let us assume that, in Figure 9b, a quasicylindrical silver electrodeposit of radius $\left\langle R_{\mathrm{c}}\right\rangle$ is formed. Then, for $0.15 \leq x \leq 0.20$ at the agarose ring, it results in $\delta_{\mathrm{r}} \cong$ $0.04 \mathrm{~cm}$, a figure close to the average branch separation distance in the growth pattern. This explanation is consistent with the outward displacement of small agarose aggregates located in gel channels by the silver growth front drift, as has been recently demonstrated. ${ }^{26}$

Furthermore, when the sol/gel transition at the interface occurs, the current decay is linked to current outburst cycles due to the local sudden outward displacement of accumulated agarose clusters at the growth front that promotes an instantaneous, local increase in the cathode surface area. Accordingly, current outbursts can be described as local stochastic pinning/ depinning cycles, the intensity of which decays with $t$, as seen in Figures 4a, 12a, and 13. This fact also contributes to further local triggering of secondary branching and pattern asymmetry.

4.3. Scenarios for Silver Electrodeposition in AgaroseContaining Media. To account for the influence of pinning on silver pattern formation, two limiting scenarios for low and high electrodeposition rates, respectively, are advanced. The first scenario (Figure 14a) leads to silver patterns that are formed by following lower resistance paths with negligible disturbance of either the percolated gel structure or the spatial distribution of small agarose aggregates. The second scenario (Figure 14b) comprises silver pattern formation in gelled supporting electrolytecontaining plating baths, in which silver electrodeposition is controlled by diffusion and advection. ${ }^{37,41}$ Consequently, the silver front drift increases $x$ at the electrochemical interface, hindering the process. For both scenarios, the contribution of screening depends on the concentration of agarose at the interface. On the basis of these scenarios, it is possible to simulate the current and charge transients and their silver pattern morphology.

4.4. Silver Pattern and Charge Transient Simulations in Colloidal Media. Silver growth pattern simulations in agarosecontaining plating baths, at constant composition and $\eta_{\mathrm{c}}$, are a)
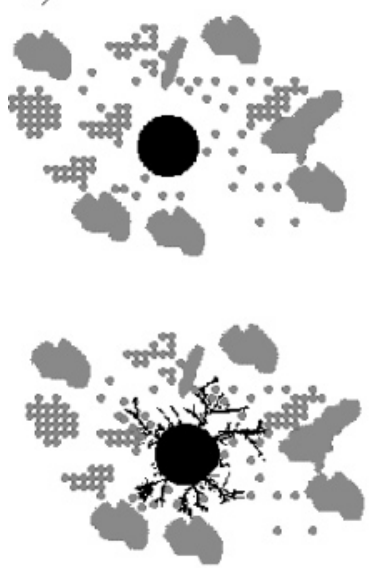

Initial working electrode
Agarose aggregates

Figure 14. Schemes for the radial growth process. The initial cathode (WE) and the agarose-containing medium (sols and gels) are indicated. (a) Slow silver pattern growth conditions imply small $\eta_{\mathrm{c}}$ without appreciable displacement of smaller agarose aggregates by the growth front. (b) Fast silver pattern growth conditions are related to large $\eta_{\mathrm{c}}$ with displacement of small agarose aggregates by the growing front. Upper schemes correspond to $t=0$. Lower schemes show silver patterns from the simulations that agree fairly well with those obtained under conditions (a) and (b).

based on the cellular automata model (CEM). ${ }^{49-51}$ The dynamics in the CEM is defined by a set of probabilistic rules that update the system in parallel. In this case, each lattice site of the CEM is occupied by either agarose species or electrodeposited silver. The application of the CEM to silver electrodeposition requires a rule for the interaction of agarose species at every surface site. The surface concentration of agarose (defined in terms of probabilities) varies between 0 (minimum coverage) and 1 (maximum coverage). The following rule governs the spreading of silver: the silver deposit grows from next-neighbor $(\mathrm{NN})$ silver sites with the probability $g=1-p$, where $p$ is the probability that a site is occupied by agarose.

In considering the above rule, an incoming silver species will try to move to a neighbor site. For assigning a probability to each site occupied by agarose, the following procedure was applied. Snapshots of agarose gel structure substrates were binarized into $N_{\mathrm{L}}$ threshold levels. Then, a probability $p=y / N_{\mathrm{L}}$ was assigned to each bin, where $y=1, \ldots, N_{\mathrm{L}}$. In this way, a high value of $p$ will correspond to those sites with a large agarose coverage. This probability distribution of agarose over all the lattice sites was used as the initial condition (Figure 15). Each simulation was started by putting a small amount of silver at the center of the lattice, and the growth of the silver phase was monitored at each time step by means of $N(t)$, the number of electrodeposited silver species.

From experimental data, ${ }^{26}$ it is known that small agarose aggregates are pushed outward as the growth front and their accumulation at the outer plane of the electrochemical interface should increase the contribution of screening to the cathodic reaction. To account for this effect, the probability $p$ of the site that was occupied by the silver deposit was equally distributed between neighbor sites containing agarose. The increase in $p$ would enhance blockage of silver spreading anisotropically, i.e., in some directions.

To avoid finite size effects, simulations were carried out by using square lattices of linear size $L=500$. Then, the simulation 


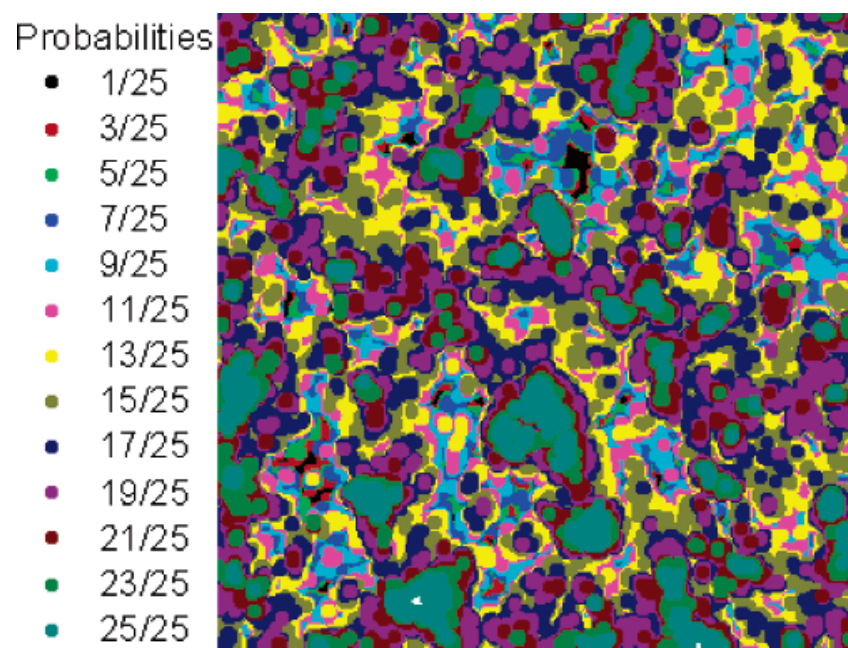

Figure 15. Image of the agarose gel built from binarized images resulting from $N_{\mathrm{L}}=25$ threshold levels. For the sake of clarity, only probabilities with odd values of $y$ are indicated in the inset.
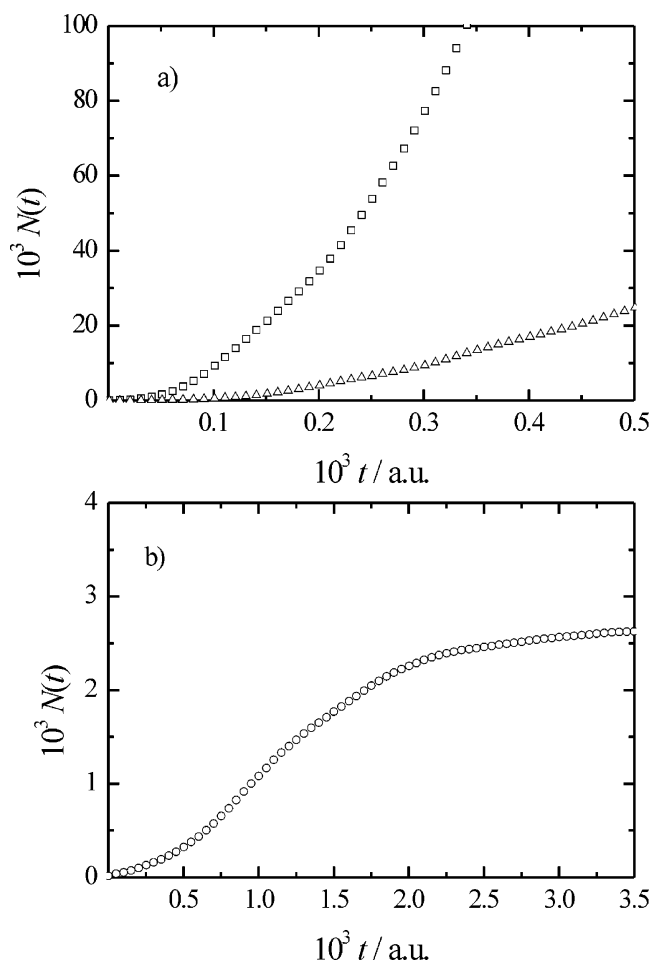

Figure 16. Results from cellular automata simulations plotted as $N(t)$ vs $t$. (a) No agarose displacement ( $\square$ ). Distribution of agarose among next- and nearest-neighbor sites $(\mathrm{NNN})(\triangle)$. (b) Distribution of agarose between next-neighbor agarose sites $(\mathrm{NN})(\mathrm{O})$. These results can be qualitatively compared to those depicted in Figures $3 \mathrm{~b}$ and $4 \mathrm{~b}$.

proceeded according to the rule mentioned above, and for the sake of comparison, three cases for agarose displacement were analyzed: (i) no agarose displacement; (ii) distribution of agarose between the next-neighbor sites (NN) that contain agarose; (iii) distribution of agarose among one next- and two nearest-neighbor sites (NNN). Further details of the algorithm as well as its limitations are given elsewhere..$^{52}$

By considering that, for silver electrodeposition, $N(t)$ is proportional to $Q_{\mathrm{c}}(t)$, simulations show that the time series of $N(t)$ (Figure 16a,b) qualitatively exhibit the same shape as the real experiments (Figures $6 \mathrm{~b}$ and 11a). For cases (i) and (iii), $N(t)$ grows indefinitely, with $t$ fulfilling a power law behavior, whereas for case (ii), a first growth regime, followed by a saturation regime, is observed (Figure 16b). The transition is due to the rule used for the distribution of agarose. In fact, for the NN distribution, the smaller number of neighbor sites makes each division of $p$ larger than for case (iii). Consequently, each division of $p$ is larger than for the NNN case. Then, the growth patterns and current transients derived from these scenarios are qualitatively comparable to the experimental ones, as expected when taking into account the model's own limitations. ${ }^{52}$

\section{Conclusions}

1. The formation of silver patterns from agarose-containing plating baths with and without addition of supporting electrolyte and their current transient data are influenced by delocalized and localized pinning. Delocalized pinning is produced by mobile agarose aggregates in sols and gels and by the accumulation of agarose aggregates caused by the silver front drift. Localized pinning is produced mainly by the percolated structure of gels. Pinning effects affect the macroscopic mass transport phenomena in the bulk. A depinning/pinning transition is observed as $x$ is increased.

2. In addition, colloidal aggregate-cathode surface interactions to some extent interfere in the attachment of discharged silver to the metal phase lattice, although the electron-transfer step in silver ion discharge seems to remain insensitive to this screening effect.

3. Both the pattern morphology and the corresponding electrochemical features are determined by the cooperative effect resulting from pinning and screening. Data from supporting electrolyte-free and supporting electrolyte-containing plating baths are analyzed as representative limiting situations. In the former case, the radial, open branched pattern morphology gradually changes from a small number of needlelike branches to an increasing number of denser branches as $x$ is increased, whereas for the latter, radial dense branched patterns are obtained, their apparent density being almost constant.

4. Local, relatively fast, pinning/depinning cycles that can be related to current fluctuations of mobile agarose aggregates at the cathode/colloidal interface introduce a random noise in the current transients. This perturbation at the microscopic scale depends on the local value of $x$, which becomes a pinningdependent variable.

5. Pattern morphologies and electrochemical data are qualitatively simulated with a simple cellular automaton model in which pinning and screening effects are mimicked via the timedependent probability of silver ion attachment to the cathode.

Acknowledgment. This work was financially supported by the Consejo Nacional de Investigaciones Científicas y Técnicas (CONICET) and PICT 98 no. 06-03251 from Agencia Nacional de Promoción Científica y Tecnológica of Argentina; M. A. Pasquale is thankful for the financial support from the Universidad Nacional de La Plata and Pontificia Universidad Católica Argentina; G. Saracco is thankful for the financial support from Fundación Antorchas, Buenos Aires, Argentina.

\section{References and Notes}

(1) Stauffer, D. Fractals and Disordered Systems, 2nd ed.; Bunde, A., Havlin, S., Eds.; Springer-Verlag: Berlin, Heidelberg, New York, 1996; Chapter 9, pp 351-360.

(2) de Boer, R. J.; van der Laan, J. D.; Hogeweg, P. Thinking about Biology; Varela, F. J., Stein, W. D., Eds.; Addison-Wesley: New York, 1992.

(3) Borman, S. Chem Eng. News 2004, 82 (51), 53-61.

(4) Yan, H.; Park, S. H.; Finkelstein, G.; Reif, J. H.; LaBean, T. H. Science 2003, 301, 1882

(5) Seeman, N. C. Sci. Am. 2004, 290, 64. 
(6) Sapoval, B. Fractals and Disordered Systems, 2nd ed.; Bunde, A., Havlin, S., Eds.; Springer-Verlag: Berlin, Heidelberg, New York, 1996; Chapter 6, pp 233-261.

(7) Barabasi, A.-L.; Stanley, H. E. Fractal Concepts in Surface Growth; Cambridge University Press: Cambridge, 1995; p 108.

(8) Sundstrom, L. G.; Bark, F. H. Electrochim. Acta 1995, 40, 599.

(9) Yamaki, J.-I.; Tobishima, S.-I.; Hayashi, K.; Saito, K.; Nemoto, Y.; Arakawa, M. J. Power Sources 1998, 74, 219.

(10) Kerr, J. B.; Han, Y. B.; Liu, G.; Reeder, C.; Xie, J.; Sun, X. Electrochim. Acta 2004, 50, 235.

(11) Winston, D.; Arora, M.; Maselko, J.; Gaspar, V.; Showalter, K. Nature 1991, 351, 132.

(12) Hassell, M. P.; Comins, H. N.; May, R. M. Nature 1991, 353, 255. 97.

(13) Rohani, P.; May, R. M.; Hassell, M. P. J. Theor. Biol. 1996, 181,

(14) Rubio, M.; Edwards, C. A.; Dougherty, A.; Gollub, J. P. Phys. Rev. Lett. 1989, 63, 1685.

(15) Buldyrev, S. V.; Barabasi, A.-L.; Caserta, F.; Havlin, S.; Stanley,

H. E.; Vicsek, T. Physica A 1992, 191, 220.

(16) Zhang, J.; Zhang, Y.-C.; Alstrom, P.; Levinsen, M. T. Physica A 1992, 189, 383.

(17) Vicsek, T.; Cserzo, M.; Horvath, V. K. Physica A 1990, 167, 300

(18) Matsushita, M.; Fijikawa, H. Physica A 1990, 168, 498.

(19) Balankin, A. S.; Susarrey, O.; Márquez González, J. Phys. Rev. Lett. 2003, 90, 096101-1.

(20) Herrasti, P.; Ocón, P.; Vázquez, J.; Salvarezza, R. C.; Vara, J. M.; Arvia, A. J. Phys. Rev. A 1992, 45, 7440.

(21) Matsushita, M.; Hayakawa, Y.; Sawada, Y. Phys. Rev. A 1985, 32,3814 .

(22) Losa, G. A.; Baumann, G.; Nonnemacher, T. F. Pathol., Res. Pract. 1992, 188,680 .

(23) Bru, A.; Pastor, J. M.; Fernaud, I.; Bru, I.; Melle, S.; Berenguer, C. Phys. Rev. Lett. 1998, 81, 4008.

(24) Bru, A.; Subiza, A. S.; López García-Asenjo, J. L.; Bru, I. Biophys. J. 2003, 85, 2948.

(25) Bru, A.; Subiza, A. S.; López García-Asenjo, J. A.; Bru, I. Phys. Rev. Lett. 2004, 92, 23101.

(26) Pasquale, M. A.; Marchiano, S. L.; Arvia, A. J. J. Phys. Chem. B 2004, 108, 9720 .

(27) Gerischer, H.; Tischer, R. P. Z. Elektrochem. 1957, 61, 1159

(28) Porter, J.; Robinson, T. O. J. Phys. Chem. 1993, 97, 6696.

(29) Pasquale, M. A.; Marchiano, S. L.; Schilardi, P. L.; Salvarezza, R.C.; Arvia, A. J. Phys. Rev. E 2002, 65, 041608.

(30) Carro, P.; Marchiano, S. L.; Hernández Creus, A.; González, S.; Salvarezza, R. C.; Arvia, A. J. Phys. Rev. E 1993, 84, R2374.
(31) Pasquale, M. A.; Marchiano, S. L.; Bolzán, A. E.; Arvia, A. J. J. Appl. Electrochim. 2003, 33, 431.

(32) Bockris, J. O'M.; Razumney, G. Fundamental Aspects of Electrocrytallization; Plenum Press: New York, 1967.

(33) Mandelbrot, B. B.; The Fractal Geometry of Nature; Freeman: New York, 1957.

(34) Schilardi, P. L.; Marchiano, S. L.; Salvarezza, R. C.; Hernández Creus, A.; Arvia, A. J. J. Electroanal. Chem. 1991, 431, 81.

(35) Newman, J. S. Electrochemical Systems, 2nd ed.; Prentice Hall: Englewood Cliffs, New Jersey, 1991.

(36) Marchiano, S. L.; Arvia, A. J. Comprehensive Treatise of Electrochemistry; Yeager, E., Bockris, J. O’M., Conway, B. E., Eds.; Plenum Press: New York and London, 1983; Vol. 6, Chapter 2, p 100.

(37) Barkey, D. P. J. Electrochem. Soc. 1990, 137, 1655.

(38) Barkey, P. D. Advances in Electrochemical Science and Engineering; Alkire, R. C., Kolb, D. M., Eds.; J. Wiley-VHC-Verlag: New York, Frankfurt a/M, 2002; p 156.

(39) Pasquale, M. A.; Marchiano, S. L.; Arvia, A. J. J. Electroanal. Chem. 2002, 532, 255.

(40) Pasquale, M. A.; Barkey, D. P.; Arvia, A. J. J. Electrochem. Soc. 2005, 152, C149.

(41) Pasquale, M. A.; Marchiano, S. L.; Vicente, J. M.; Arvia, A. J. J. Phys. Chem. B 2004, 108, 13315.

(42) Avnir, D. The Fractal Approach to the Heterogeneous Chemistry;

J. Wiley: New York, 1989; Chapter 5.

(43) Tang, L.-H.; Leschhorn, H. Phys. Rev. A 1992, 45, R8309.

(44) Buldyrev, S. V.; Barabasi, A.-L.; Caserta, F.; Havlin, S.; Stanley,

H. E.; Viscek, T. Phys. Rev. A 1992, 45, R8313.

(45) Sneppen, K. Phys. Rev. Lett. 1998, 69, 3833.

(46) Bort, A. H.; Juettner, K.; Lorenz W. J.; Schmidt, E. J. Electroanal. Chem. 1977, 84, 127.

(47) Schmidt, E.; Siegenthaler, H. J. Electroanal. Chem. 1983, 150, 59.

(48) Damaskin, B. B.; Petrii, O. A.; Batrakov, V. V. Adsorption of Organic Compounds on Electrodes; Plenum Press: New York, 1971; p 238.

(49) Wolfram, S. Theory and Applications of Cellular Automata; World Scientific: Singapore, 1986.

(50) Solé, R. V.; Manrubia, S. C. Orden y Caos en Sistemas Complejos; Universitat Politécnica de Catalunya: Barcelona, 1996; p 125.

(51) Stauffer, D. Fractals and Disordered Systems, 2nd ed.; Bunde, A. Havlin, S., Eds.; Springer-Verlag: Berlin, Heidelberg, New York, 1996; Chapter 9.

(52) López, A. C.; Saracco, G. P.; Albano, E. V. Europhys. Lett. 2000, 52,8 . 